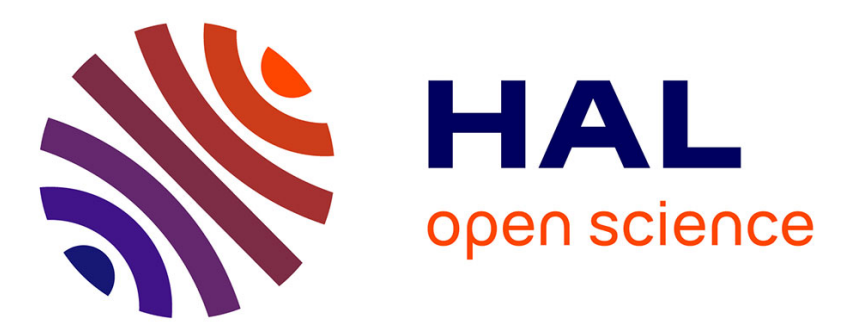

\title{
A new numerical strategy for SPF pressure profile computing based on statistical strain rate controlling
}

Maxime Rollin, Luc Penazzi, Vincent Velay, Alain Dupuy, Sylvie Gallet

\section{To cite this version:}

Maxime Rollin, Luc Penazzi, Vincent Velay, Alain Dupuy, Sylvie Gallet. A new numerical strategy for SPF pressure profile computing based on statistical strain rate controlling. International Journal of Mechanical Sciences, 2018, 141, p.479-490. 10.1016/j.ijmecsci.2018.04.021 · hal-01781300

\section{HAL Id: hal-01781300 https://imt-mines-albi.hal.science/hal-01781300}

Submitted on 6 Jun 2018

HAL is a multi-disciplinary open access archive for the deposit and dissemination of scientific research documents, whether they are published or not. The documents may come from teaching and research institutions in France or abroad, or from public or private research centers.
L'archive ouverte pluridisciplinaire HAL, est destinée au dépôt et à la diffusion de documents scientifiques de niveau recherche, publiés ou non, émanant des établissements d'enseignement et de recherche français ou étrangers, des laboratoires publics ou privés. 


\title{
A New Numerical Strategy for SPF Pressure Profile Computing Based on Statistical Strain Rate Controlling
}

\author{
M. Rollin ${ }^{1,2, *}$, L. Penazzi ${ }^{1}$, V. Velay ${ }^{1}$, A. Dupuy ${ }^{2}$, S. Gallet ${ }^{2}$ \\ ${ }^{1}$ Institut Clément Ader (ICA), Université de Toulouse, CNRS, Mines Albi, INSA, UPS, ISAE, \\ Campus Jarlard, 81013 Albi CT Cedex 09, France \\ ${ }^{2}$ AIRBUS Operations S.A.S., 316 Route de Bayonne, 31060 Toulouse Cedex 09, FRANCE
}

\begin{abstract}
This paper investigates the designing of Superplastic Forming pressure cycles in order to propose rapid forming sequences that suit industrial strategy, and which enable the control of microstructure evolutions. An optimization algorithm using ABAQUS ${ }^{\circledR}$ coupled with MATLAB ${ }^{\circledR}$ has been developed in order to improve forming cycles while allowing superplasticity. The numerical strategy is based on an algorithm controlling a statistical maximum of the distribution of strain rate of nonformed elements of part mesh instead of the maximum strain rate on the whole mesh for classical optimization algorithms. The objective of this method is to exclude numerical singularities that are used for pressure controlling in traditional techniques. This paper details firstly the new algorithm and then presents numerical and experimental results for different forming conditions obtained from industrial, classical, and optimized pressure cycles. These tests were performed on Ti-6Al-4V titanium alloys.
\end{abstract}

Keywords: Superplastic forming, Finite Element Modeling, Optimization, Strain Rate Distribution 


\begin{tabular}{|ll|}
\hline$\dot{\varepsilon}_{\text {opt }}$ & Optimal strain rate \\
$\varepsilon_{c r i t}$ & Critical deformation \\
$\varepsilon_{c r i t}^{+}$ & Very critical deformation \\
$S_{t h}$ & Thickness of part \\
$X^{i}$ & Quantity referring to an increment $i$ \\
$X^{n}$ & Quantity referring to a step $n$ \\
$X^{k}$ & Quantity referring to the $k^{\text {th }}$ iteration of a step \\
$<X>$ & Average of $X$ quantity \\
$\widehat{s}(X)$ & Standard deviation of $X$ quantity \\
$\mathcal{R}$ & Strain rate ratio for pressure increment \\
$\mathcal{S}$ & Relative standard deviation \\
$\underline{\Delta}_{A-B}$ & Relative difference of $X_{A}$ versus $X_{B}$ \\
\hline
\end{tabular}

\section{Introduction}

Superplastic forming (SPF) is a hot forming process for sheet metal. Classical SPF consists in blowing gas under pressure in to a chamber in order to form sheet metal on a mold. SPF has many advantages: it can be used to form complex shapes with a high yield rate, thin and fairly constant sheet thickness and with no significant finishing stage. However, it requires high forming temperatures, and a low strain rate compared to stamping and forging processes in general. In addition, SPF requires particular material with a specific microstructure morphology that promotes superplastic mechanisms.

The key to the SPF process is to control the pressure during forming in order to produce strain rates in a specific range that can be called the superplastic domain. By this method, the properties of superplastic materials prevent strain localization and promote rather reasonably uniform part thickness. This domain is defined at a constant temperature as the strain rate range that allows high elongation. The domain is dependent on microstructure because the deformation mechanisms asso- 
ciated with superplasticity (grain boundary sliding, and the accommodation diffusional flow mechanisms) are dependent on grain size and morphology [1].

A lot of work has been done to reduce forming time over recent decades because of the necessity to improve this time-consuming process. A first axis of improvement concerns the material properties, and the objective is to move the superplastic domain. Mohamed et al. [2] highlighted that reducing the material grain size caused an elevation of the superplastic strain rate range. Since their study, a large body of work has been undertaken on so-called ultrafine grain microstructures, which not only increase the strain rate range, but also lower the temperature of the superplastic domain. Figueiredo et al. [3] achieved a strain rate ten times higher with $0.2 \mu \mathrm{m}$ grain size, obtained by an equal-channel angular pressing process with an aluminum alloy. Matsumoto et al. [4] obtained low temperature with high strain rate superplasticity on a Ti-6al-4V alloy produced by simple hot working of an $\alpha^{\prime}$-martensite microstructure alloy. One problem of these grain size refining processes is that they are difficult to implement industrially. A second axis of improvement concerns the process conditions. The pressure is adjusted in order to form as fast as possible. Regardless of the superplastic domain, it is important to stay inside its range to ensure the maximum elongation properties.

Finite Element modeling is widely used in the industry to define the appropriate pressure cycle. Optimization of a pressure cycle resides in controlling the pressure during forming so that the strain rate of the part stays within an acceptable range. In this paper, only the design of the pressure cycle is studied, for a fixed temperature and initial microstructure. The author has developed a statistical approach for the evaluation of the strain rate field, and especially the maximum. The maximum strain rate is the usual value used to regulate pressure, and ultimately define a pressure cycle. The objective of using statistics is to obtain field distribution information, and therefore to exclude numerical singularities in order to obtain physical data. The author's method makes it possible to select appropriate information to improve the forming cycle.

However, there are some possible consequences of increasing global strain rate during SPF. Firstly, one important problem is that strain rate sensitivity decreases when 
the strain rate increases. Alabort et al.[1] illustrated this phenomenon with a contour map of the strain rate sensitivity exponent $m$ as a function of temperature and strain rate. The consequence is that thinning or necking of the part is likely to happen prematurely. Gifkins [5] investigated the interactions between grain boundary sliding and the accommodation mechanisms. The consequence of increasing strain rate is that GBS is not accommodated, resulting in void growth. Finally, dynamic grain growth is strain rate sensitive. The grain size increase disrupts the grain boundary sliding mechanisms (the main superplastic mechanism), as mentioned by Langdon [6]. Velay et al. [7] have shown that the grain size is significantly impacted by time exposure and strain rate conditions during superplastic deformation.

In the following sections, the different existing numerical strategies for pressure designing are summarized. Then the authors' method is presented, applied first on a standard geometry, and secondly on an industrial aircraft workpiece. The SPF of the pressure cycle obtained on the industrial part using this new method is compared to that of a "classical method". The previously cited impacts on material and geometry are analyzed in the last part of the paper.

\section{Background}

Finite Element (FE) modeling of SPF was introduced in the 1980's by Bellet [8] to address the need to improve the thickness profile, surface and mechanical properties of parts, but also to adapt the pressure profile to increase the forming rate while allowing superplasticity. Globally, pressure cycle optimization is based on two different aspects: a reference quantity to target, and a pressure algorithm to control pressure so as to form at the targeted quantity throughout the forming process. The reference quantity is usually an optimal strain rate $\dot{\varepsilon}_{\text {opt }}$ defined as the highest strain rate for which the strain rate sensitivity exponent $m$ is superior to a critical value. Indeed, $m$ (defined in Equation (1)) is a marker of superplasticity. Woodford [9] has pointed out that for some metals, high elongation properties imply a high $m$ value.

$$
m=\frac{\partial \ln (\sigma)}{\partial \ln (\dot{\varepsilon})}
$$


- Reference quantity

There are different scalar quantities that can be used for forming, but the strain rate is mostly the preferred choice. Garriga-Majo [10] compared the strain rate, the flow stress and the energy dissipation rate as quantities to target during simulation. Their results indicated that the energy dissipation rate value gave the best results in terms of forming time, but to the detriment of surface quality. The material model can be coupled with grain size evolution and void growth, which are both major disruptive phenomena for the stability of superplastic deformation. The value of $\mathrm{m}$ and therefore $\dot{\varepsilon}_{\text {opt }}$ evolves throughout the forming process. Ding et al. [11] developed a variable strain rate path. They used the gradient of the strain rate over the sheet, which has the advantage of preserving local thinning. Nazzal et al. [12] used a variable strain rate approach (similarly to Ding et al.) to perform the SPF simulation of a rectangular box and an industrial aircraft part. They compared a constant with a variable strain rate approach and the forming time reduction was found to be significant. Chung and Cheng developed [13] and experimented [14] a strain sensitive optimization of pressure through a so-called flow localization factor (FLF). This FLF is derived from the instability analysis of Hart [15], and makes it possible to take advantage of the excellent necking resistance of superplastic materials. This method led to a considerable improvement in forming time compared to constant or even variable strain rate paths, but gave a less uniform thickness distribution in the tested case.

- Pressure algorithms

Robert has summarized [16] three main types of algorithms. Firstly, there are the proportional corrective algorithms developed initially by Doltsinis [17]. They consist of a continuous function which can take account of different parameters like the control quantity, material parameters, etc. Since Doltsinis' work, a large number of increment functions have been developed with more or less complexity. For instance, Xiang et al. [18] used a function of the relative difference of targeted and maximum strain rates, whereas Hwang et al. [19] 
used a function of the logarithm of the ratio of both quantities. Then, Bellet [8] developed the band algorithm which consists in using different ranges of strain rate ratio values around one, where each range is linked to a constant corrective

pressure factor. Finally, there are the post-treatment methods proposed by Carrino et al. [20]. The technique consists in running a simulation with a known constant pressure cycle, and then correcting the pressure by adjusting the associated maximum strain rate during forming. This type of algorithm requires two simulations, but is easy to implement.

Each improvement cited above enables forming to be completed as fast as possible, thus reducing the forming time. However, the basic principle of these numerical strategies is based on a single element among thousands of others, and sometimes more. This method would work if FE simulation could ensure that perfect continuous stress and strain rate fields were obtained, which, by definition, is not the case. Obviously this hypothesis is not the optimal one for two main reasons, namely contact solving issues and mesh singularities. Indeed, the contact algorithms can lead to stress discontinuities due to penetration and interference solving. Typically, for the passage of the sheet on a small curvature radius, these problems are frequent (Figure 1). Moreover, large deformations can lead to distorted elements, even if the initial mesh has been structured and adapted to the major strain axis. These elements regularly produce singularities in the stress field. The principle of the method developed in this paper is to free the pressure cycle optimization from these discontinuities which lead to an overestimation of the strain rate ratio and limit the forming time.

\section{Optimization technique}

There are two important aspects in this method that differ from the classical strategies presented in section 2. The first one consists in replacing the classical maximum strain rate $\dot{\varepsilon}_{\max }$ that is used in the strain rate ratio. The term to be used here will be called the maximum of the distribution of strain rate $\dot{\varepsilon}_{\text {max }}^{i}$. The second difference resides in the pressure incrementation. In order to be industrially feasible 


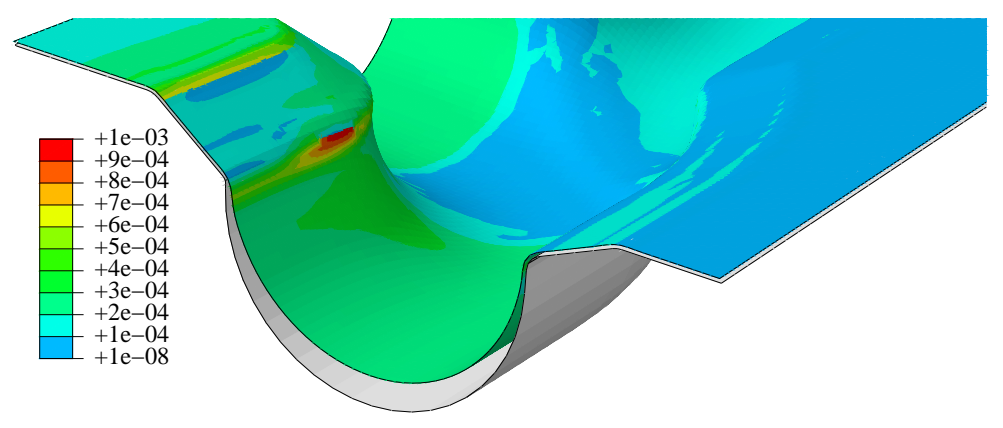

(a)

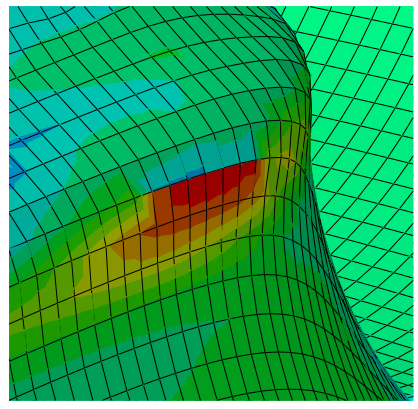

(b)

Figure 1: (a) Strain rate field during SPF simulation of a part, (b) zoom on discontinuous elements

(and also to limit numerical iterations), the pressure is incremented considering not every increment but the integration of the strain rate distribution over a specific step time. Both aspects are detailed hereafter. Henceforth, the presented method will be called the "Strain Rate Distribution-based" (SRD) method, in opposition to the classical one, the "Local Maximum Strain Rate-based" method (LMSR).

\subsection{Statistical Maximum}

The solution retained to determine a maximum from the strain rate field is to model it by a Gaussian distribution. Therefore, it is possible to fix a maximum by knowing the average and the standard deviation of the strain rate field. However, the shape of the distribution does not fit a normal-centered distribution insofar as the part is formed, because more and more elements get in contact and deformed at a limited rate due to friction shear stresses. The second assumption made is to select only the elements which are not in contact for the computation of the distribution of strain rate. The selection is made by a contact pressure criterion. This is an easy way to avoid the problem and also to reduce the forming time by focusing only on the high strain rate elements. This hypothesis is valid because the key point is to stay below the optimal strain rate.

$$
\begin{gathered}
\mathcal{P}(X<\mu+2 \sigma)=\int_{-\infty}^{\mu+2 \sigma} \frac{1}{\sigma \sqrt{2 \pi}} e^{\left.-\frac{1}{2}\left(\frac{X-\mu}{\sigma}\right)^{2}\right)} d X \simeq 0.98 \\
\mu=\frac{1}{N_{\Omega}} \sum_{k \in \Omega} X_{k} \\
\sigma=\sqrt{\frac{1}{N_{\Omega}} \sum_{k \in \Omega}\left(X_{k}-\mu\right)^{2}}
\end{gathered}
$$


Gaussian theory says that for a population (or a set of elements) which has an associated data, $98.1 \%$ of the elements have their data below the average data of the set of elements plus twice its standard deviation. This probability is defined in Equation (2) where $\mathcal{P}, \mu$ and $\sigma$ represent respectively the density probability function of $\mathrm{X}$, the mean value and the standard deviation of $\mathrm{X}$.

$$
\dot{\varepsilon}_{G M}=\mu+2 \sigma
$$

Applying this technique to the strain rate field, the singular elements can be excluded or at least, their number taken in the computation of $\dot{\varepsilon}_{\max }^{i}$ will be limited. For the rest of the paper, the maximum of strain rate distribution will be called the "Gaussian Maximum" (GM) of strain rate field $\dot{\varepsilon}_{G M}$ and is defined by Equation (5). Figure $2 \mathrm{a}$ represents the comparison of the histogram density $\left(\%_{e l}\right)$, the associated "Normalized Probability Density Function" (NPDF) for the "Whole mesh" (Wh) and the "non-contact" (NC) set of elements $\Omega_{N C}$. $\Omega_{N C}$ is defined by Equation (6) where $\Omega_{W h}, P c_{e l}, k, P^{i}$ are respectively the set of all elements of the part, the contact pressure of an element, a pondering coefficient equal to 0.2 and the forming pressure. The data was taken from an increment of the SPF simulation from which Figure 1 was extracted. The NPDF data was related to the total number of elements to highlight the proportion of elements between the whole mesh and the selected elements. This figure clearly confirms a best fit of data in the case where non-contact elements are taken compared to the whole mesh at this forming stage

$$
\Omega_{N C}=\left\{e l \in \Omega_{W h} \mid P c_{e l}>k P^{i}\right\}
$$

Three maximums are represented in Figure 2a, the gaussian maximums of both probability functions $\dot{\varepsilon}_{G M}^{W h}$ and $\dot{\varepsilon}_{G M}^{N C}$, and the "Local Maximum" $\max _{e l \in \Omega_{W h}}\left(\dot{\varepsilon}_{e l}\right)$ (written $\left.\max \left(\dot{\varepsilon}_{e l}\right)\right) . \quad \dot{\varepsilon}_{G M}^{W h}$ is considerably higher than the local maximum, which indicates that the Gaussian distribution is not efficient in this case. On the contrary, $\dot{\varepsilon}_{G M}^{N C}$ is below the local maximum. Figure $2 \mathrm{~b}$ is a zoom of Figure $2 \mathrm{a}$ in the high strain rates area. The $\%_{e l}$ of whole mesh set is split by $\dot{\varepsilon}_{G M}^{N C}$ value into $W h^{-}$and $W h^{+}$sets to highlight the elements excluded by the method. It reveals that the elements which are excluded by this technique correspond to a small percentage of all the elements 


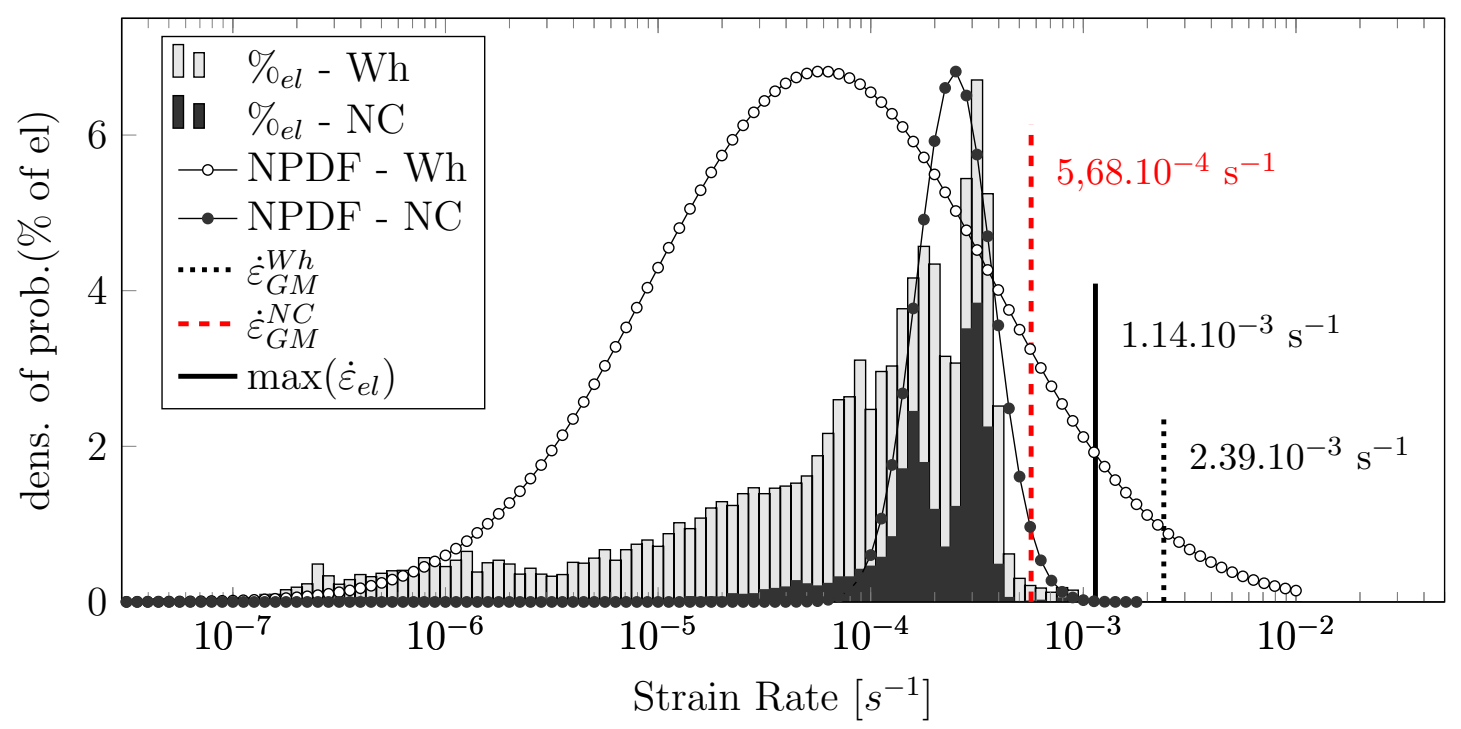

(a)

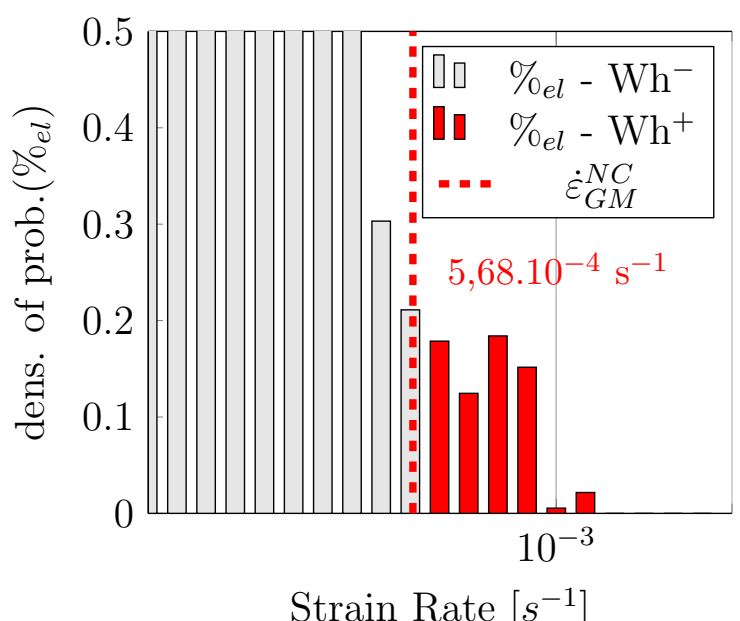

(b)

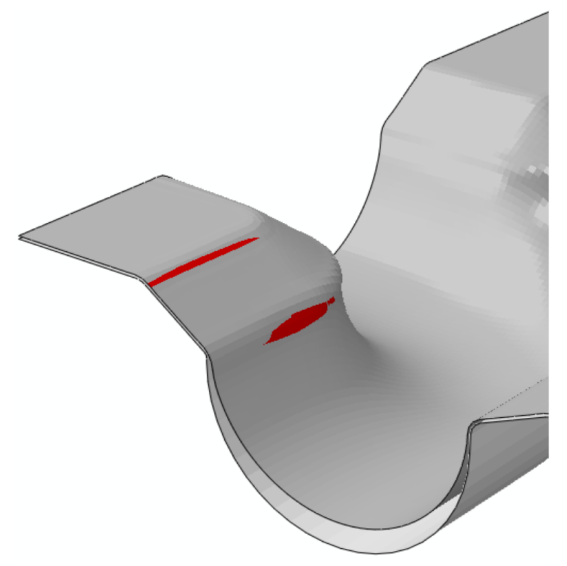

(c)

Figure 2: illustration of statistical strain rate controlling - (a) Distribution, normal probability function and maximums of Strain rate field for both Wh and NC sets of elements at Figure 1 increment, (b) zoom of (a) graph around $\dot{\varepsilon}_{G M}^{N C}$ value and c) representation of excluded elements on part mesh

$(0.67 \%)$. Figure 2c represents these excluded elements on the part. By comparing these elements with their associated strain rate in Figure 1, it is interesting to observe that they correspond to discontinuous elements. To conclude, this method is reasonably efficient for selecting only the elements that are relevant to fit the distribution of strain rate and finally compute its maximum. 


\subsection{Pressure incrementation}

As mentioned previously (in section 2), the pressure is corrected over a step and not at each increment in the simulation. For each increment that the FE software computes, the average strain rate $\left\langle\dot{\varepsilon}_{e l}>^{i}\right.$ and the standard deviation of the distribution of strain rate $\widehat{s}\left(\dot{\varepsilon}_{e l}\right)^{i}$ are computed. $\left\langle\dot{\varepsilon}_{e l}>^{i}\right.$ and $\widehat{s}\left(\dot{\varepsilon}_{e l}\right)^{i}$ are defined in equations (7) and (8), where $\dot{\varepsilon}_{e l}, i$ and $\Omega^{i}$ are respectively the equivalent strain rate of an element, the increment number and the set of the non-contact elements $\Omega_{N C}$ at the increment $i$ whose size is $N_{\Omega_{i}}$.

$$
\begin{gathered}
<\dot{\varepsilon}_{e l}>^{i}=\frac{1}{N_{\Omega_{i}}} \sum_{e l \in \Omega_{i}} \dot{\varepsilon}_{e l} \\
\widehat{s}\left(\dot{\varepsilon}_{e l}\right)^{i}=\sqrt{\frac{1}{N_{\Omega_{i}}} \sum_{e l \in \Omega_{i}}\left(\dot{\varepsilon}_{e l}-<\dot{\varepsilon}_{e l}>^{i}\right)^{2}} \\
\Omega^{i}=\left\{e l \in \Omega_{W h} \mid P c_{e l}>k P^{i}\right\}
\end{gathered}
$$

Then, the maximum of the distribution of strain rate of an increment $\dot{\varepsilon}_{\text {max }}^{i}$ is computed with Equation (10). Finally, the average maximum of the distribution of strain rate of a step $\left\langle\dot{\varepsilon}_{\text {max }}^{i}>^{n}\right.$ is time-step integrated as defined in Equation (11) where $t^{i}$, $N$ and $t_{N}$ are respectively the time of increment $i$, the number of increments in step $n$ and the time of step $n$.

$$
\begin{gathered}
\dot{\varepsilon}_{\text {max }}^{i}=<\dot{\varepsilon}_{e l}>^{i}+2 \times \widehat{s}\left(\dot{\varepsilon}_{e l}\right)^{i} \\
<\dot{\varepsilon}_{\text {max }}^{i}>^{n}=\frac{1}{t_{N}} \sum_{i}^{N} t^{i} \dot{\varepsilon}_{\text {max }}^{i}
\end{gathered}
$$

This $\left\langle\dot{\varepsilon}_{\text {max }}^{i}>^{n}\right.$ value is used in the pressure algorithm instead of the local maximum for LMSR method. The authors chose to use a band algorithm for pressure incrementation. The method is composed by three cases defined in table 1 where $\mathcal{R}$, $P$ and $\alpha$ are respectively the pressure algorithm ratio defined with Equation (12) for step $n$, the pressure and the pressure incrementation factor. The cases 1 and 3 correspond to the lower $\mathcal{R}_{\text {low }}$ and upper bounds $\mathcal{R}_{\text {crit }}$ for the ratio $\mathcal{R}$ beyond which

the forming conditions of the step are considered critical. In these cases the step is restarted with a corrected pressure ramp computed with $\alpha$ coefficient. In the case 2 , the forming conditions are considered acceptable and the pressure ramp of 


\begin{tabular}{c|c|c} 
CASE & IF & THEN \\
\hline 1 & $\mathcal{R}^{n}<\mathcal{R}_{\text {low }}$ & $P^{n, k+1}=\alpha_{\text {low }} P^{n}$ \\
2 & $\mathcal{R}_{\text {low }}<\mathcal{R}^{n}<\mathcal{R}_{\text {crit }}$ & $P^{n+1}=\alpha^{n} P^{n, k}$ \\
3 & $\mathcal{R}^{n}>\mathcal{R}_{\text {crit }}$ & $P^{n, k+1}=\alpha_{\text {crit }} P^{n}$
\end{tabular}

Table 1: Pressure regulation table - definition of cases

the next step is computed with $\alpha_{n}$ pressure incrementation factor. The values used for pressure regulation are given table 9 in section 6 . However, any other pressure algorithm can be implemented.

$$
\mathcal{R}^{n}=\frac{<\dot{\varepsilon}_{\text {max }}^{i}>^{n}}{\dot{\varepsilon}_{\text {opt }}}
$$

\subsection{Implementation of the new strategy}

The SRD method is implemented on the same basis as the LMSR method (as programmed in ABAQUS ${ }^{\circledR}$ ) and presented in Figure 3a, where $i$ and $k$ are respectively the increment, the increment's iteration number and $P$ the pressure.

However, the integration on the time step of $\dot{\varepsilon}_{\text {max }}^{i}$ can produce an exceedance of $\dot{\varepsilon}_{\text {opt }}$. For example, when a portion of the sheet comes into contact with the mold, the strain rate can vary considerably. Figure 4 illustrates a typical evolution of $\dot{\varepsilon}_{\text {max }}^{i}$, which induces an exceedance of some $\dot{\varepsilon}_{\text {max }}^{i}$ values with an average $\left\langle\dot{\varepsilon}_{\text {max }}^{i}\right\rangle^{n}$ below the $\dot{\varepsilon}_{\text {opt }}$. There are three major schemes to evaluate the maximum strain rate of a step (used for pressure algorithm) from maximum of increments. The first one is to select the maximum of all increments. In this case, the problem is that the associated maximum strain rate of the increment can be influenced when high discontinuities happen during particular complex forming phase. A second solution is to apply the same statistical approach than for mesh distribution. The idea is to use the average value $\left\langle\dot{\varepsilon}_{\text {max }}^{i}>^{n}\right.$ and to compute $\widehat{s}\left(\dot{\varepsilon}_{\text {max }}^{i}\right)$ with Equation (13) where $N$ is the number of increments in the step. Then, the new ratio to consider for the pressure algorithm would be $\mathcal{R}^{n^{\prime}}$, defined in Equation (14), where twice the standard deviation is again introduced to avoid the problem. This strategy was tested first, 


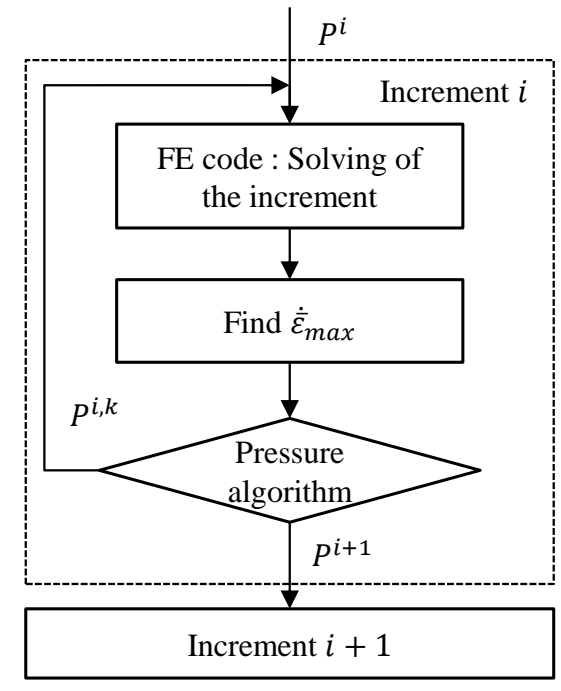

(a)

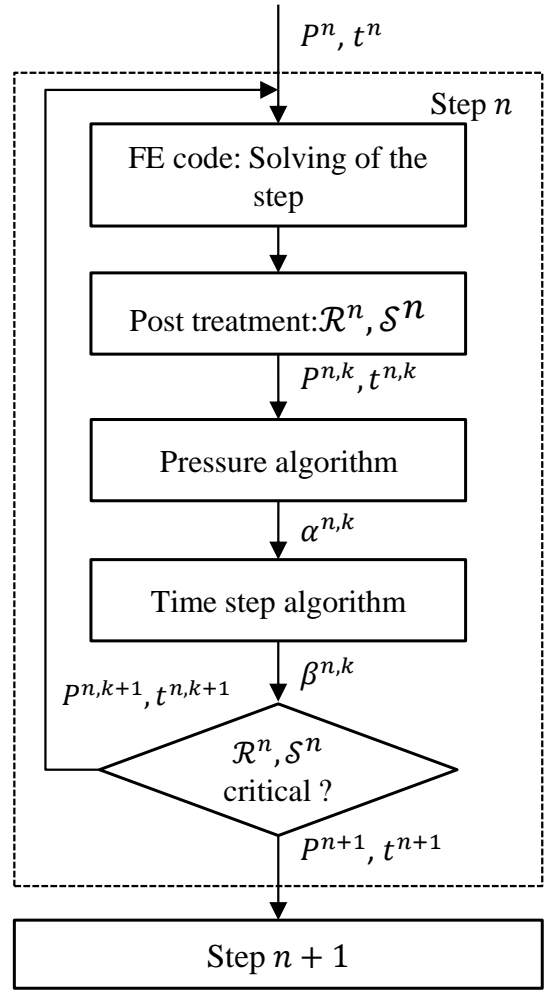

(b)

Figure 3: Logigrams of pressure algorithms implementation of: (a) classical LMSR method and (b) SRD method

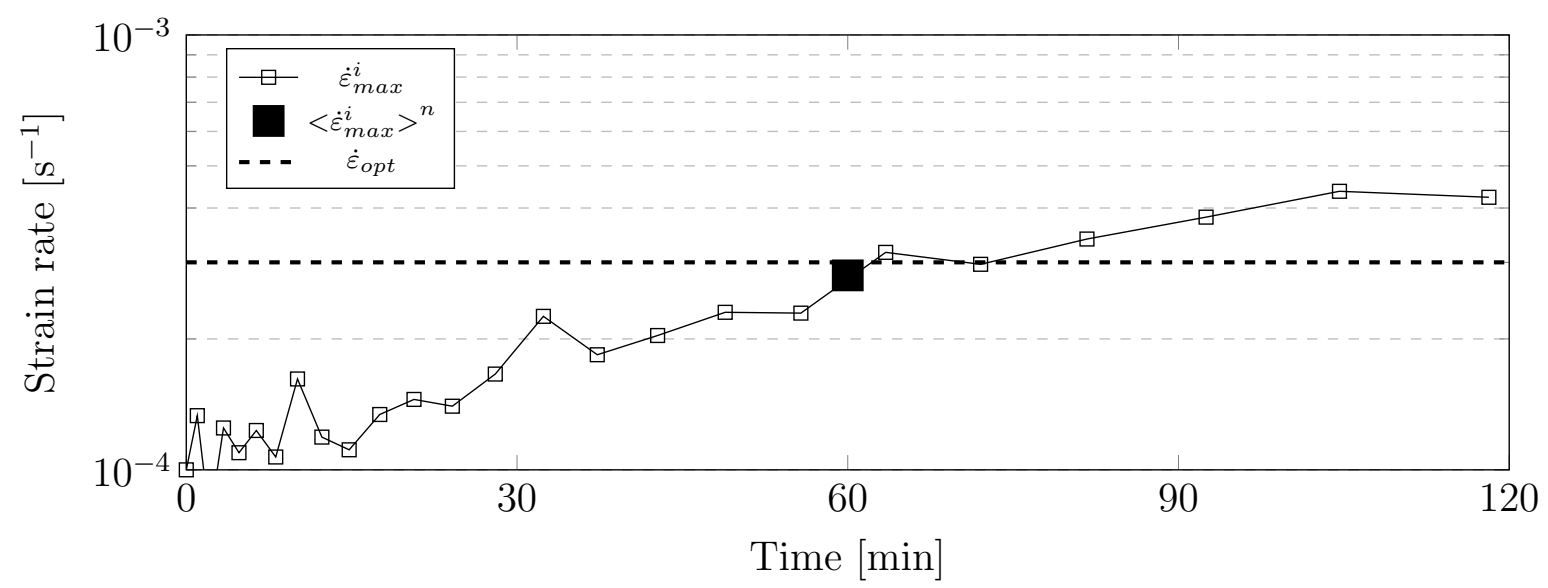

Figure 4: Example of Strain rate indicators evolution during a step at Figure 1 forming stage

and gave high oscillations of pressure and convergence difficulties.

$$
\widehat{s}\left(\dot{\varepsilon}_{\text {max }}^{i}\right)=\sqrt{\frac{1}{t_{N}} \sum_{i}^{N} t^{i}\left(\dot{\varepsilon}_{\text {max }}^{i}-<\dot{\varepsilon}_{\text {max }}^{i}>^{n}\right)^{2}}
$$




$$
\mathcal{R}^{n^{\prime}}=\frac{<\dot{\varepsilon}_{\text {max }}^{i}>^{n}+2 \widehat{s}\left(\dot{\varepsilon}_{\text {max }}^{i}\right)}{\dot{\varepsilon}_{\text {opt }}}
$$

The last solution consists in imposing a maximum variation of $\dot{\varepsilon}_{\text {max }}^{i}$ beyond what the step is not considered acceptable. When this situation is met, the step is restarted with a smaller time step in order to limit large variations of strain rate distribution. This variation of $\dot{\varepsilon}_{\max }^{i}$ is estimated thanks to the relative standard deviation $\mathcal{S}^{n}$ (Equation (15)). The time step is regulated as a function of $\mathcal{S}^{n}$ in order to prevent the $\dot{\varepsilon}_{\max }^{i}$ variation in a step becoming too large. Table 2 defines three cases which have an effect on the time step. The values used for $\mathcal{S}_{\text {low }}$ and $\mathcal{S}_{\text {crit }}$ are respectively $7.5 \%$ and $15 \%$. In the case $1, \beta_{\text {low }}$ is equal to $3 / 2$, and in the case $3, \beta_{\text {crit }}$ is equal to $2 / 3$. These values can be changed according to the geometry which influences the forming stability. The weakness of this second method is that some increments of a step can have $\dot{\varepsilon}_{\text {max }}^{i}$ superior to $\dot{\varepsilon}_{\text {opt }}$. The maximum possible strain rate $\dot{\varepsilon}_{\text {err }}$ that can be allowed is defined in Equation (16), where $\mathcal{R}_{\max }$ is the maximum acceptable ratio for SRD strategy.

$$
\begin{gathered}
\mathcal{S}^{n}=\frac{\widehat{s}\left(\dot{\varepsilon}_{\text {max }}^{i}\right)}{<\dot{\varepsilon}_{\text {max }}^{i}>^{n}} \\
\dot{\varepsilon}_{\text {err }}=\mathcal{R}_{\text {max }} \times \dot{\varepsilon}_{\text {opt }}\left(1+2 \mathcal{S}_{\text {crit }}\right)
\end{gathered}
$$

Sometimes, during SPF simulation there is no possibility to cross a step without

\begin{tabular}{c|c|c} 
CASE & IF & THEN \\
\hline 1 & $\mathcal{S}^{n}<\mathcal{S}_{\text {low }}$ & $t^{n, k+1}=\beta_{\text {low }} t^{n, k}$ \\
2 & $\mathcal{S}_{\text {low }}<\mathcal{S}^{n}<\mathcal{S}_{\text {crit }}$ & $t^{n+1}=t^{n, k}$ \\
3 & $\mathcal{S}^{n}>\mathcal{S}_{\text {crit }}$ & $t^{n, k+1}=\beta_{\text {crit }} t^{n, k}$
\end{tabular}

Table 2: Time step regulation table

getting $\mathcal{S}^{n}$ inferior to $\mathcal{S}_{\text {crit }}$. When several iterations are done for a step without getting an acceptable $\mathcal{S}^{n}$ value, $\mathcal{S}_{\text {crit }}$ is momentarily doubled to overcome the step before returning to its initial value once the step is correctly evaluated. Equation (17) gives the computation of pressure as defined in the global algorithm where the pressure is computed before the time step adjustment.

$$
P^{n+1}=\frac{P^{n+1}-P^{n}}{t^{n}} t^{n+1}+P^{n}=\left(\left(\alpha^{n}-1\right) \beta^{n}+1\right) P^{n}
$$


This procedure was implemented with ABAQUS ${ }^{\circledR}$ FE software, MATLAB ${ }^{\circledR}$ (for procedure automation) and Python scripts (for the post treatments), as described in Figure 3b. MATLAB ${ }^{\circledR}$ manages the creation of step job files and the restarting from previous jobs until the fulfillment of several conditions to detect the end of the forming process.

\subsection{Numerical Validation: Rectangular box}

\subsubsection{Model}

In this section, the proposed method is implemented and compared with a model that has already been used in the literature. This model is the rectangular box used by Nazzal et al. [12] to develop a variable strain rate approach. The box is $60 \mathrm{~cm}$ long by $40 \mathrm{~cm}$ wide by $20 \mathrm{~cm}$ deep with a $2 \mathrm{~cm}$ flange around it, and the sheet metal is $3.175 \mathrm{~mm}$ thick. A Norton Hoff power law was selected for use in material modeling (Equation (18)). $K$ and $m$ coefficients were obtained from mechanical testing characterization at forming temperature and strain rate range $(K=3210, m=0.66)$. This model is not strain sensitive, which is in contradiction with the definition of a variable strain rate path (VSRP). However, it has been used numerically for comparison purposes. The author's method is independent of pressure algorithms, and can also be combined with other optimization techniques. In the first step, the presented method is compared with the classical LMSR method proposed by ABAQUS ${ }^{\circledR}$, for three different schemes. The two first schemes consist in forming at a constant targeted strain rate at $10^{-4} s^{-1}$ and $10^{-2} s^{-1}$, regulated on

the whole mesh. The third scheme consisted in forming using the VSRP by following the element with the maximum strain. The VSRP was developed by Nazzal et al. [12], and corresponds to the stability analysis with no initial void fraction (see Figure 5). In the second step, a geometry with a smaller fillet radius (from $40 \mathrm{~mm}$ to 6 $\mathrm{mm}$ ) was tested to promote hard contact conditions and numerical singularities to demonstrate the effectiveness of the method on a geometry closer to an industrial one. Both geometries are shown in Figure 6. Shell elements were used to model the mold and sheet. They were composed respectively of 423 and 445 rigid shell elements R3D4, and by 704 and 2816 deformable shell elements S4R for large and small radius 
fillets. A surface-to-surface contact algorithm was defined between sheet and mold using a Coulomb friction law with a 0.1 coefficient value.

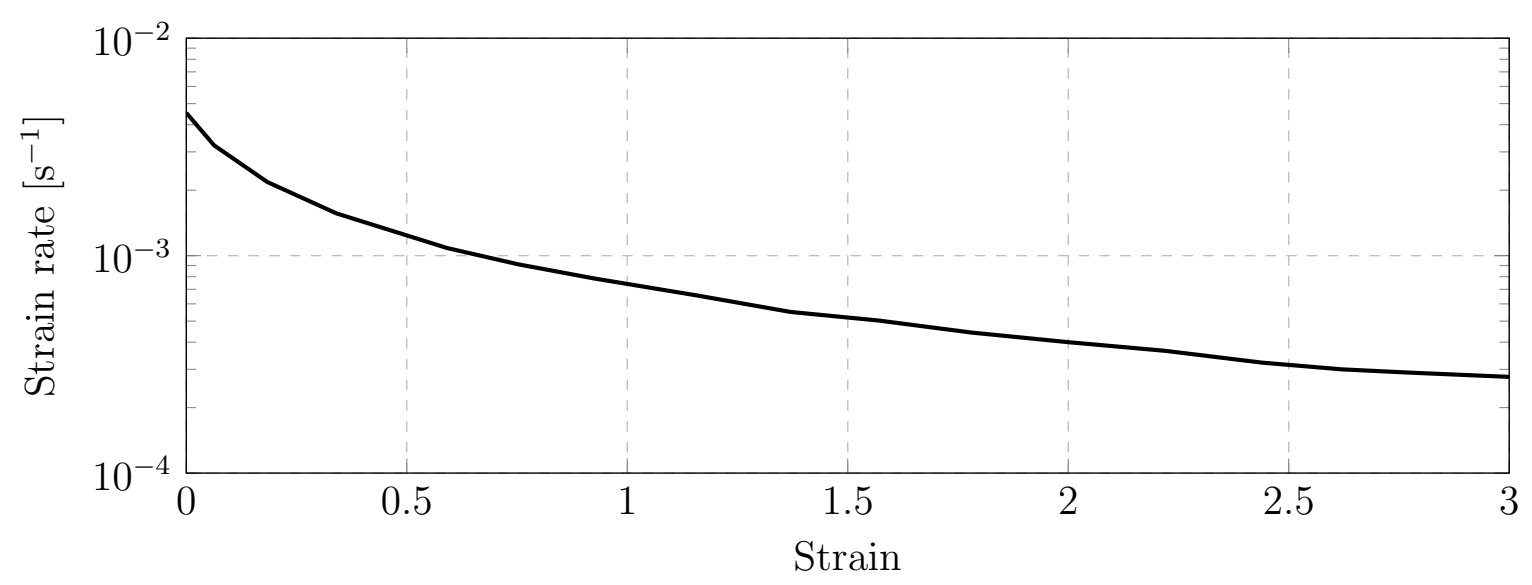

Figure 5: Variable optimal strain rate path with no initial void fraction [12]

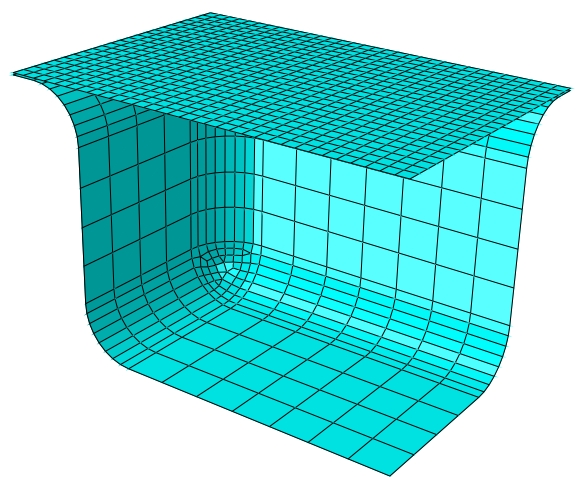

(a)

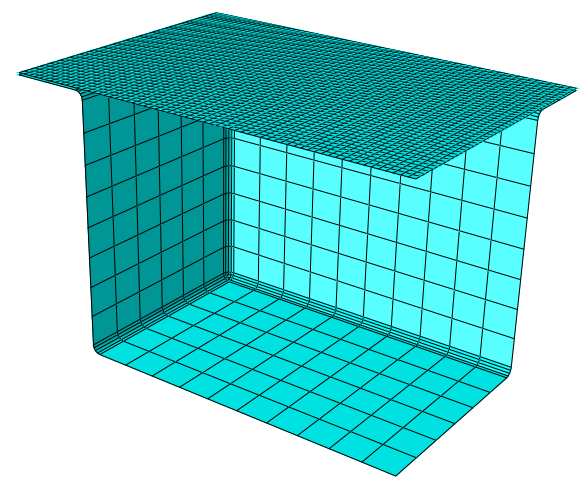

(b)

Figure 6: Meshed model of rectangular boxes with (a) large and (b) small fillet radius

$$
\sigma=K \dot{\varepsilon}^{m}
$$

\subsubsection{Results}

The pressure cycles obtained for the initial and for the second geometry are presented for each of the three schemes in Figure 7 and Figure 9. A comparison of the strain rate indicators $\max _{e l \in \text { mesh }}\left(\dot{\varepsilon}_{e l}\right)$ (written $\left.\max \left(\dot{\varepsilon}_{e l}\right)\right)$ and $\dot{\varepsilon}_{\text {max }}^{i}$ are presented for SRD pressure cycle simulations respectively in Figure 8 and Figure 10. The differences between LMSR and SRD pressure cycles and strain rate indicators are 
larger for the second geometry with the small fillet radius. Indeed, there are many discontinuities especially for the simulation with $\dot{\varepsilon}_{\text {opt }}=10^{-4} \mathrm{~s}^{-1}$ (Figure 10a). This confirms the efficiency of the method when contact conditions are severe.

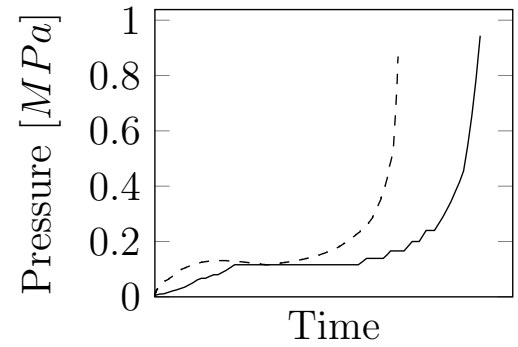

(a)

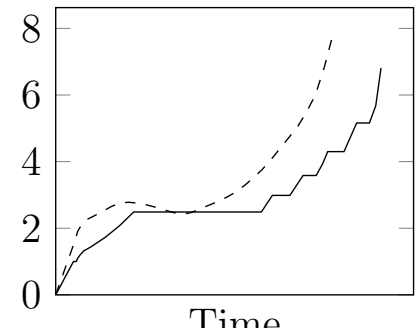

Time

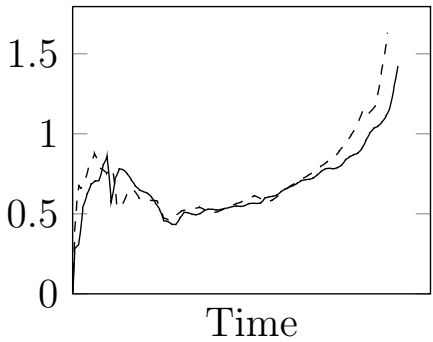

(c)

Figure 7: Comparison of pressure cycles obtained with LMSR (solid line) and SRD (dashed line) method (a) $\dot{\varepsilon}_{\text {opt }}=10^{-4} \mathrm{~s}^{-1}$, b) $\dot{\varepsilon}_{\text {opt }}=10^{-2} \mathrm{~s}^{-1}$ and c) VSRP) with large fillet radius geometry

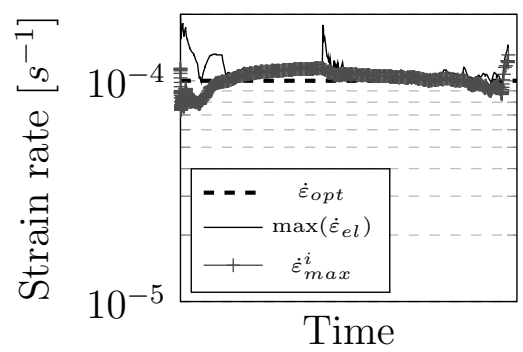

(a)

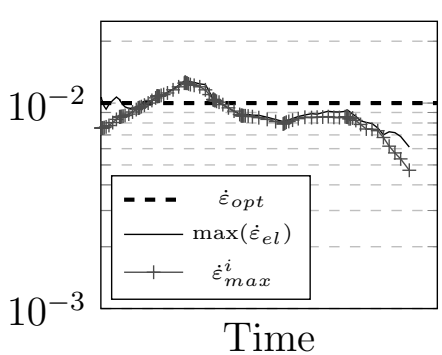

(b)

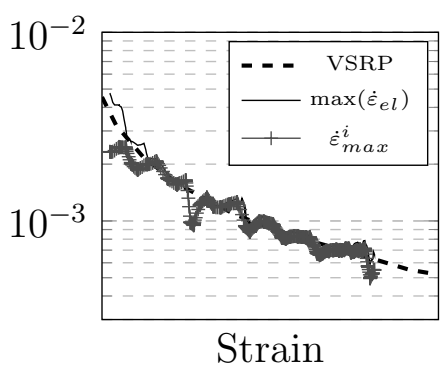

(c)

Figure 8: Evolution of strain rate indicators during forming of large fillet radius with a) $\dot{\varepsilon}_{\text {opt }}=10^{-4}$ $\left.\mathrm{s}^{-1}, \mathrm{~b}\right) \dot{\varepsilon}_{\text {opt }}=10^{-2} \mathrm{~s}^{-1}$ and c) VSRP

$$
\Delta_{A-B}=\frac{X_{A}-X_{B}}{X_{A}}
$$

Equation 19 defines for the rest of the paper the relation of relative difference of $X_{A}$ and $X_{B}$ where $X_{B}$ is the reference or initial value of the comparison.

Table 3 summarizes the forming times obtained with the different schemes and methods. A first conclusion is that the author's method clearly decreases the forming times without significantly increasing the pressures. A further conclusion is that the second geometry, with a small radius, gives better results in terms of forming time, especially for $\dot{\varepsilon}_{\text {opt }}=10^{-2} \mathrm{~s}^{-1}$ and VSRP. This is mainly due to the contact conditions 


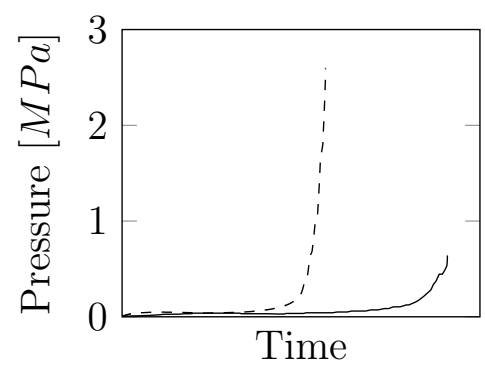

(a)

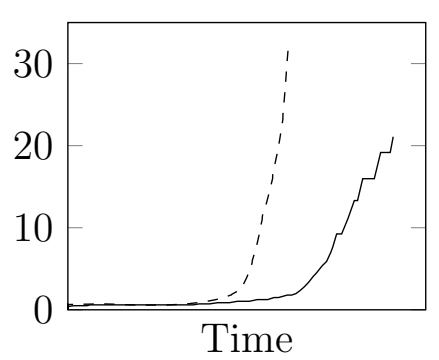

(b)

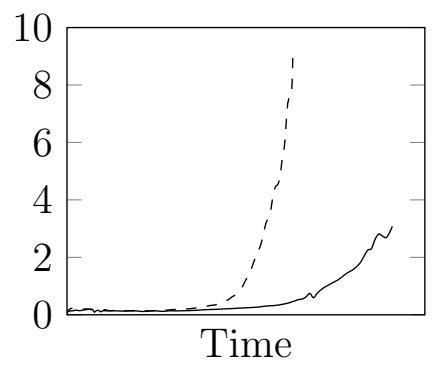

(c)

Figure 9: Comparison of pressure cycles obtained with LMSR (solid line) and SRD (dashed line) method (a) $\dot{\varepsilon}_{o p t}=10^{-4} \mathrm{~s}^{-1}$, b) $\dot{\varepsilon}_{\text {opt }}=10^{-2} \mathrm{~s}^{-1}$ and c) VSRP) with small fillet radius geometry

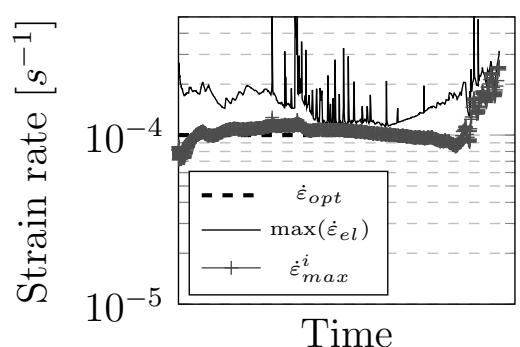

(a)

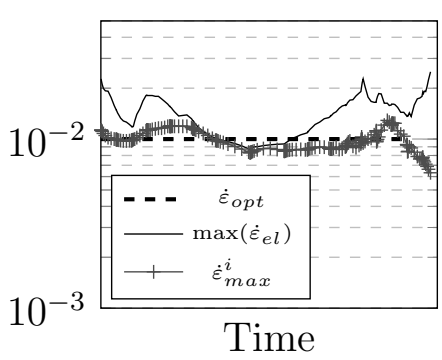

(b)

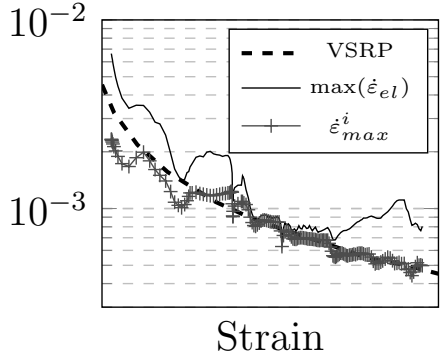

(c)

Figure 10: Evolution of strain rate indicators during forming of small fillet radius with a) $\dot{\varepsilon}_{\text {opt }}=$ $10^{-4} \mathrm{~s}^{-1}$, b) $\dot{\varepsilon}_{\text {opt }}=10^{-2} \mathrm{~s}^{-1}$ and c) VSRP

\begin{tabular}{c|c|c|c||c|c|c} 
& $10^{-4} s^{-1}$ & $10^{-2} s^{-1}$ & VSRP & $10^{-4} s^{-1}$ & $10^{-2} s^{-1}$ & VSRP \\
\hline $\operatorname{LMSR}(\mathrm{s})$ & 19551 & 183 & 1620 & 30479 & 318 & 4100 \\
\hline SRD (s) & 14619 & 165 & 1557 & 19070 & 215 & 2849 \\
\hline$\Delta t_{L M S R-S R D}(\%)$ & 25 & 15 & 4 & 37 & 32 & 31 \\
\hline
\end{tabular}

Large Radius

Small Radius

Table 3: Comparison of Forming Times

that are harder in these case, and thus, promote much singularities that are excluded by the authors strategy. After post-treating the results it appears that most of the elements which overshooted during the simulation were numerical singularities, such as those presented in Figure 2c. However, some elements undergo real deformation and can be excluded by the method. In the next section, microstructure and geometrical analyses are presented to interpret the exclusion of these critical elements 
for the forming of an industrial part.

\section{Validation of the method: Industrial workpiece}

\subsection{Simulation of the case}

This method was applied to optimize the forming cycle of an AIRBUS A350 part. This workpiece is half of a T-duct in Ti-6Al-4V alloy (Figure 11a). The initial thickness of the blank was around $3.2 \mathrm{~mm}$. A chemical milling (1.5 mm depth) was applied in the center area to generate a pocket. This enabled to promote material deformation to be facilitated in this area and limited the thinning of the sheet in the useful portion of the part. This detail is an important issue and will be discussed later to point out the limitations of certain hypotheses of this strategy.

A quarter of the part was modeled (Figure 11b). A VISCO step was used with material model described in previous section 3.4. The blank had 18741 quadrilateral shell elements with a reduced integration (S4R) and the mold had 3524 rigid quadrilateral shell elements (DS4). The contact was modeled with a "surface-to-surface" algorithm with a finite sliding approach. The Coulomb friction law was chosen with a coefficient of 0.1 .

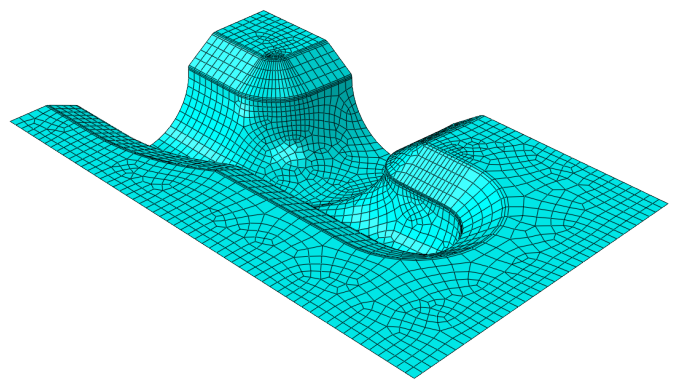

(a)

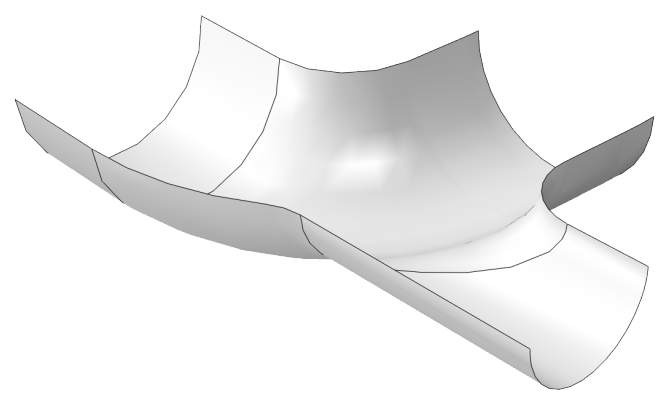

(b)

Figure 11: Representation of (a) The mold meshed and (b) the 3D CAD of final part after trimming

In addition to the strain rate distribution based method, the classical LMSR method was computed in order to provide a reference to compare with. The results of the SRD method (which are presented later) come from a whole simulation computed with the complete pressure cycle instead of the pressure step files obtained from 
initial computing. As regards the CPU time issues for solving complete SPF, SRD methods spent only one and a half times more than LMSR methods (Table 4).

\begin{tabular}{c|c|c} 
CPU Time (min) & LMSR & SRD \\
\hline ABAQUS $^{\circledR}$ & 2014.4 & 3056.8 \\
MATLAB $^{\circledR}$ \& Python & 0 & 9.6 \\
Total & 2014.4 & 3066.4
\end{tabular}

Table 4: Comparison of numerical solving time for both methods

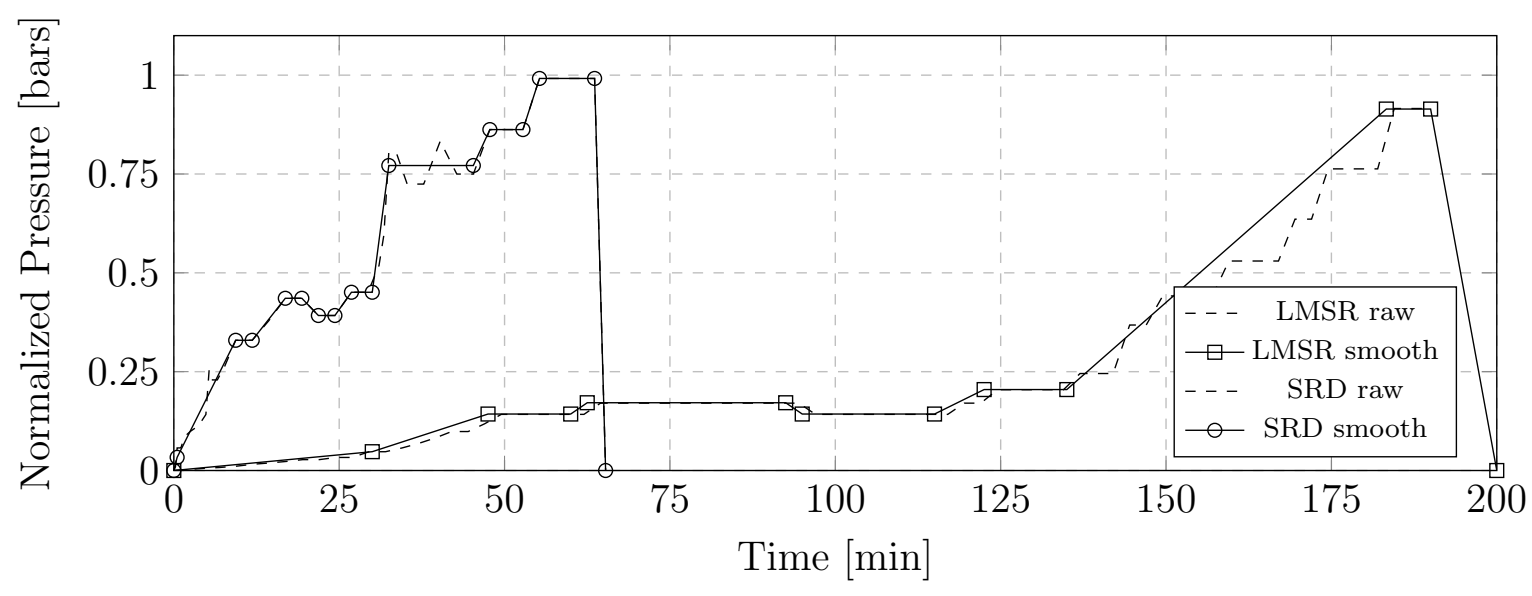

Figure 12: Comparison of pressure cycles obtained by LMSR and SRD based optimization methods

The pressure implementation cycles obtained with the LMSR and SRD methods are presented in Figure 12. In order to remove numerical artifacts like pressure oscillation, to simplify the cycle and also to ease implementation in the press automate, the curves have been smoothed. The SRD method has greatly improved the forming time. It has been divided approximately by three compared with the forming time for LMSR simulation (from 200 to $67 \mathrm{~min}$ ). The forming time saving is particularly high for this geometry due to the pocketing surface that lead the pressure profile for LMSR strategy. This case is very specific and enables to test the limits of the method in terms of consequences on material and geometrical specifications. Some other industrial geometries such as air inlet of reactors, and other fairing parts of the pylon were tested regarding to those two numerical strategies. The global time saving is approximately around 30 to $50 \%$ of reduction according to geometries. 


\subsection{Critical deformation}

The objectives of performing SPF simulation with both pressure cycles numerically obtained are to analyze the impact on the formability of the part, on the final thickness, and the microstructure. Hence, to validate the model, microstructural analysis must be carried out on sample cuts on formed parts. A critical deformation field was computed (Equation (20)) to target different areas to investigate. This field (Figure 13) was only computed for the SRD method because the LMSR method has no critical deformation by definition.

$$
\begin{aligned}
& \varepsilon_{c r i t}=\int_{\dot{\varepsilon}>\dot{\varepsilon}_{o p t}} \dot{\varepsilon} d t \\
& \varepsilon_{c r i t}^{+}=\int_{\dot{\varepsilon}>\dot{\varepsilon}_{e r r}} \dot{\varepsilon} d t
\end{aligned}
$$

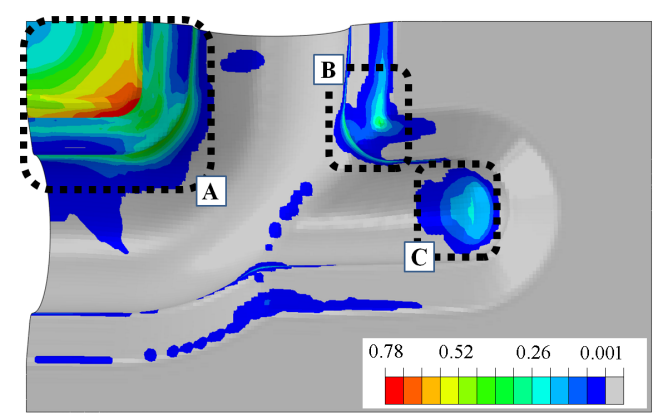

(a)

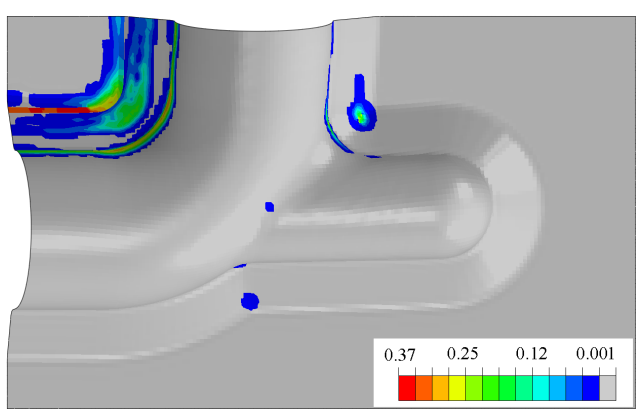

(b)

Figure 13: Iso-values of (a) critical deformation $\varepsilon_{\text {crit }}$ with marked areas and (b) very critical deformation $\varepsilon_{c r i t}^{+}$

The critical strain rate field represented in Figure 13a highlights three main areas where the part has deformed over the optimal strain rate:

- Zone A: the most important critical deformation can be divided into two portions. The first corresponds to the initially pocketed area of the blank (see Figure 13b, the blue zone). Logically this surface played its role and has not been taken into account by the SRD method (elements in contact with the mold). The second portion is the rest of the zone. These elements have critical strain due to the passage of a "hard" contact area of the mold with a small radius of curvature and a strong bi-directional deformation in the corner of the pocketed area. 
- Zone B: this zone is also due to "hard" contact conditions

- Zone C: this zone is constituted by elements which did not follow the hard contact conditions. They are the last-formed elements.

In order to verify the impact of this computed critical deformation, six samples, called observation points, were selected, as defined in Figure 14. Point 1 has the

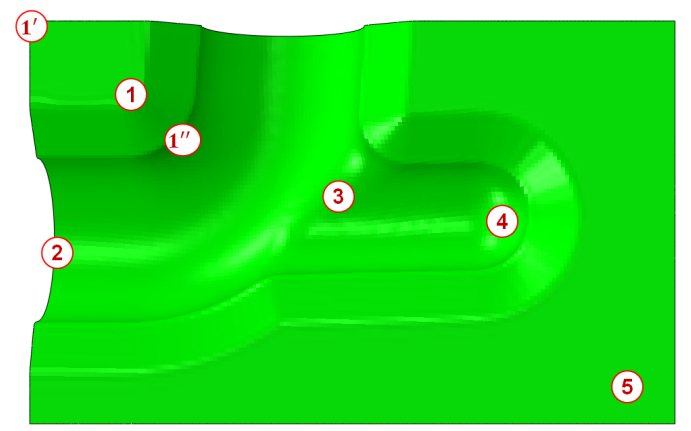

Figure 14: Location on the part of observation points

maximum critical strain. A second critical strain field, as defined in Equation (21), was computed in order to evaluate the elements that were deformed in very critical strain rate conditions compared to what can be called semi-critical strain (between $\dot{\varepsilon}_{\text {opt }}$ and $\left.\dot{\varepsilon}_{e r r}\right)$. Point 1 is one of the only points that was deformed in very critical conditions (Figure 13b). Point 2 corresponds to an area with mid-strain level and no critical deformation. Point 3 corresponds to a low strain level with a small critical deformation. Point 4 is included in Zone $\mathrm{C}$, with high strain level and critical deformation without contact issues. Finally, point 5 is a control observation which did not undergo deformation. Points $1^{\prime}$ and $1^{\prime \prime}$ are specific points only used for thickness measurements, described later.

\subsection{Experimental validation}

Two full-size parts were formed. One with an LMSR and the other with an SRD pressure cycle (Figure 12). Both cycles were capable of forming the parts completely. Figure 15 shows one of the formed part. Different parameters, such as thicknesses and microstructure issues, were investigated. Samples were cut (according to Figure 14) to observe either microstructure aspects, or thickness impacts, or both. 


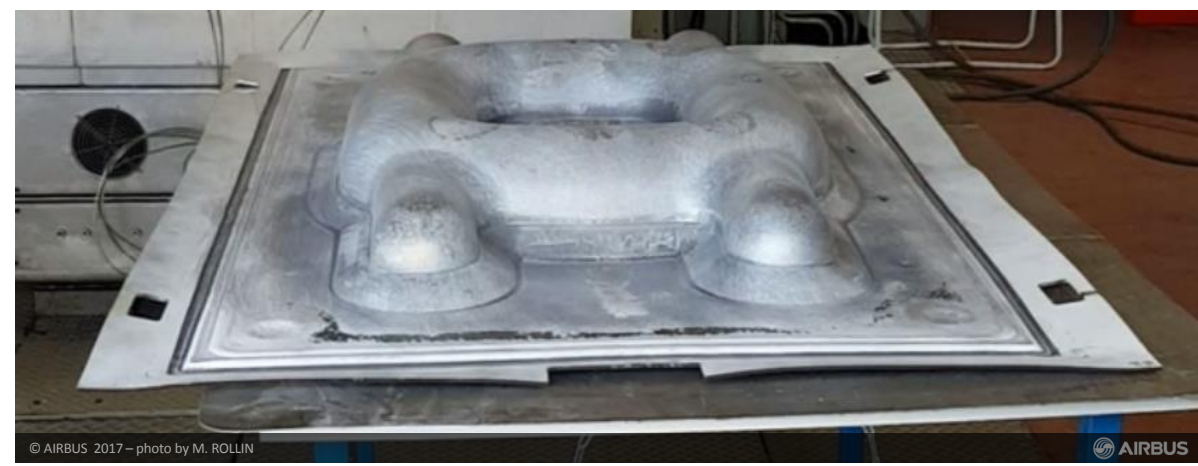

Figure 15: View of a workpiece after forming

\subsubsection{Thickness impact}

Thickness measurements have been performed on both parts in areas shown in Figure 14. Point 1 has not been retained because in this area, the thickness is discontinuous due to pocketing operation, and the tolerances around this area have promoted excessive variations of thicknesses. Points $1^{\prime}$ and $1^{\prime \prime}$ have been selected instead for this highly deformed area. Thickness measurements have been performed by ultrasound mean on experimental formed parts. The measurements have been quadrupled (measured on each quarter of part) for each position. Table 5 presents a comparison of the results obtained by measurement and by simulation.

\begin{tabular}{|c|c|c|c|c|c|}
\hline & \multicolumn{5}{|c|}{ Point position } \\
\hline & $1^{\prime}$ & $1^{\prime \prime}$ & 2 & 3 & 4 \\
\hline \multicolumn{6}{|c|}{ LMSR } \\
\hline$S t h^{E x p}(\mathrm{~mm})$ & 1.28 & 1.83 & 2.27 & 2.4 & 1.64 \\
\hline$S_{t h}^{\text {Sim }}(\mathrm{mm})$ & 1.13 & 1.97 & 2.35 & 2.62 & 1.71 \\
\hline$\underline{\Delta} \operatorname{Sth}_{\operatorname{Exp}-\operatorname{Sim}}(\%)$ & 11.5 & -7.9 & -3.5 & -9.2 & -4.2 \\
\hline \multicolumn{6}{|c|}{ SRD } \\
\hline$S t h^{\operatorname{Exp}}(\mathrm{mm})$ & 1.31 & 1.85 & 2.23 & 2.31 & 1.62 \\
\hline$S_{t h}{ }^{\text {Sim }}(\mathrm{mm})$ & 1.17 & 2.04 & 2.35 & 2.65 & 1.73 \\
\hline$\underline{\Delta} \operatorname{Sth}_{\operatorname{Exp}-\operatorname{Sim}}(\%)$ & 10.8 & -10.0 & -5.5 & -14.7 & -6.8 \\
\hline
\end{tabular}

Table 5: Comparisons of thicknesses of simulation and measurement at some observation points

Firstly, when comparing the experimentation with the simulation, it is clear that 
for Point $1^{\prime}$ the thicknesses of the model are more than ten percent lower than experiment for both strategies while all other points have higher thicknesses with the model. This phenomenon can have different origins. One can be the strain rate sensitivity used in the material model. It may not reproduce precisely enough the behavior and a dependency to strain rate could probably improve the results. An other reason can be the pocketing operation that would have not been performed at the tolerance, which has conducted to a limitation of material flow in this region and affected the rest of the part. Table 5 gives the relative thickness differences for experimentation that were pondered by the ratio of nominal (Sth $=3.2 \mathrm{~mm}$ ) and experimental initial thicknesses $\left(S t h_{I N I}^{L M S R}=3.27 \mathrm{~mm}\right.$ and $\left.S t h_{I N I}^{S R D}=3.27 \mathrm{~mm}\right)$ as defined by Equation 22.

$$
\widetilde{S t h}^{E x p}=S t h^{E x p} \times \frac{S t h_{0}}{S t h_{I N I}}
$$

Then, the comparison of pondered experimental thicknesses shows that there is no significant impact of the strategy for the measured points.

Point position

\begin{tabular}{c|c|c|c|c|c} 
initial Sth & $1^{\prime}$ & $1^{\prime \prime}$ & 2 & 3 & 4 \\
\hline$\underline{\Delta \widetilde{S t h}_{L M S R-S R D}(\%)}$ & 1.1 & 0.14 & -3.04 & -5.17 & -2.57 \\
\hline
\end{tabular}

Table 6: Relative differences of thickness measurements pondered by initial value between LMSR and SRD forming strategies at some observation points

\subsubsection{Microstructure evolution}

Microstructure analysis issues. Before analyzing the microstructure of samples cut from the formed part, it is important to understand the phase transformation mechanisms that occur during cooling, and what parameters can influence these transformations. At SPF temperature, in Ti-6Al-4V, there are two phases, $\alpha$ and $\beta$, at their equilibrium content. During cooling, the temperature varies very fast and consequently $\alpha$ and $\beta$ phase proportions also vary. Ahmed and Rack [21] have shown that below the beta transus at low, moderate and high cooling rates (between 0 and $30{ }^{\circ} \mathrm{C} . \mathrm{s}^{-1}$ ), the transformation of the beta phase can lead to three different 
transformations. The appearance of these transformations depends principally on the cooling rate. In our case, when the part is removed from the mold, there is a first sequence where the part is cooled down naturally, and also forced convections. The part is also affected by radiative heat transfers which vary along the complex geometry and, finally, the thickness of sheet is not constant. For all these reasons, the cooling rate of the part is totally heterogeneous, and not constant along the cooling sequence, which induces heterogeneous phase transformations. Therefore, simple qualitative comparisons are made hereafter concerning grain morphology, and grain size comparisons are made sample by sample.

SEM micrographs. Micrographs of samples cut on both formed parts were performed with a NOVA nanoSEM 450 Scanning Electron Microscope (Figure 16 with $\times 2000$ magnification factor). The micrographs were performed with back scattered electron detector, at $8.0 \mathrm{kV}$ beam voltage, $5.0 \mathrm{~mm}$ working distance with a beam deceleration mode which bring topological details of the surface. Table 7 summarizes thickness, total strain and critical deformation of each point that was observed. Globally, the first difference that can be observed between LMSR and SRD micrographs is the grain size, and this point is discussed in the next paragraph. Secondly, as explained in the previous paragraph, each point has a specific microstructure. When comparing sample by sample, there is no significant difference in the morphology of the grains. No texturing, cavities or other consequences of rapid deformation outside of superplastic domains can be observed in the micrographs. Sample 1 has deformed in very critical conditions (see at point 1 position in Figure 13 and 14) with SRD pressure cycle, but no differences are revealed with the LMSR sample. For all the other points, the conclusions are identical.

\begin{tabular}{c|c|c|c|c|c} 
Point & 1 & 2 & 3 & 4 & 5 \\
\hline$S_{\text {th }}(\mathrm{mm})$ & 0.8 & 2.3 & 2.6 & 1.7 & 3.2 \\
$\varepsilon_{\text {crit }}$ & 0.77 & 0 & 0.01 & 0.2 & 0 \\
$\varepsilon_{\text {tot }}$ & 0.84 & 0.41 & 0.3 & 0.74 & 0
\end{tabular}

Table 7: Thickness, critical strain and total strain at observation points 

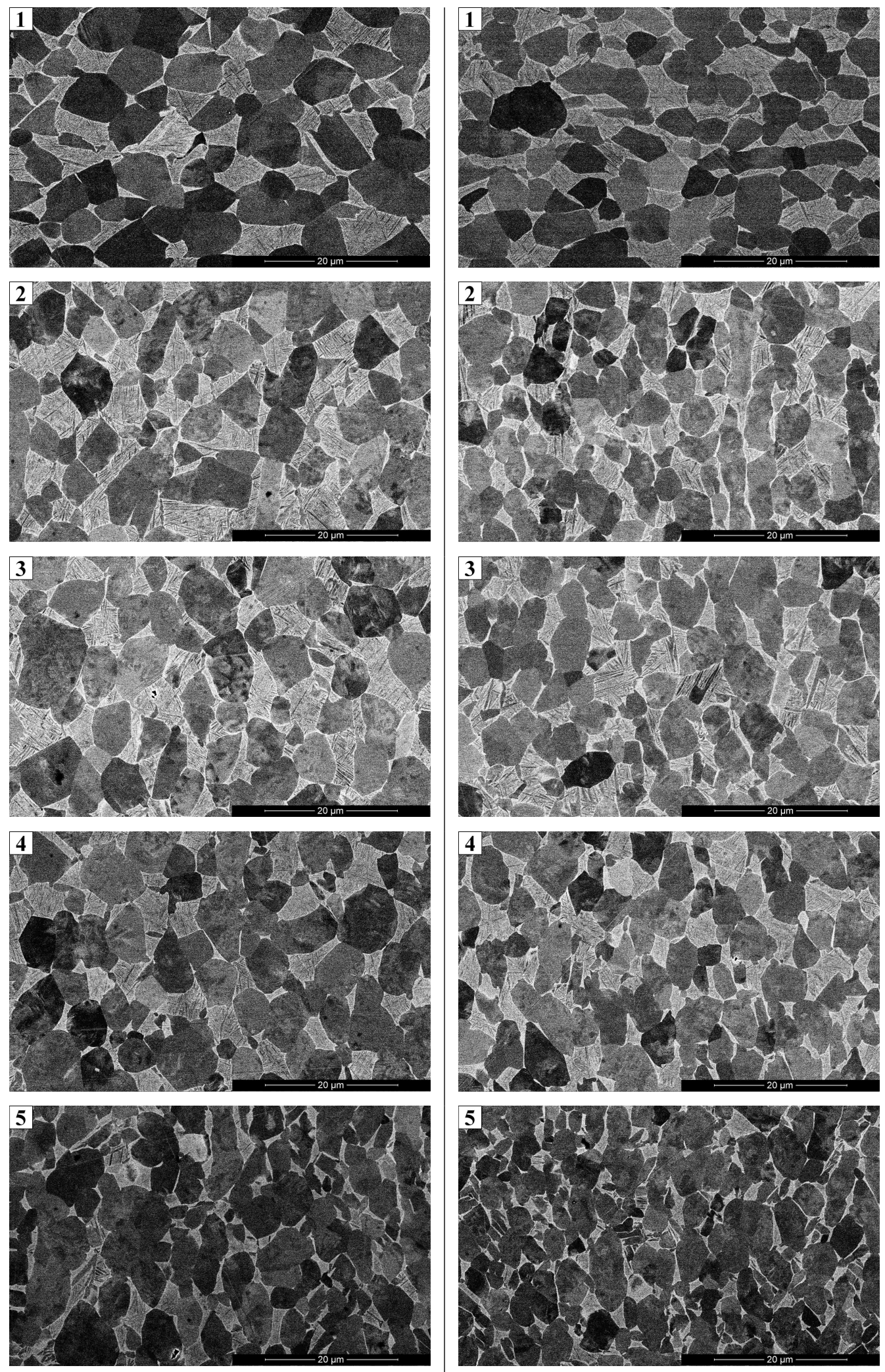

LMSR

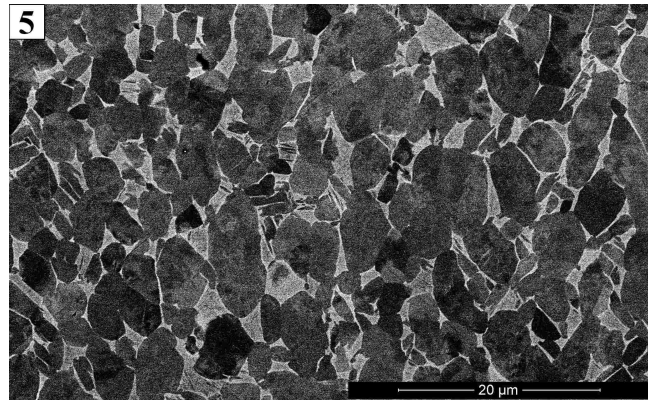

SRD

Figure 16: Micrographs of observation points defined in Figure 14 at x2000 magnification for LMSR and SRD methods 
Grain growth issues. The grain size (GS) is also an important parameter of the microstructure characteristics at the end of forming, for several reasons. Indeed, aeronautical standards impose a maximum GS to respect material specifications. In addition, the GS influences the properties of the material in terms of strain hardening and yield strength. It can be clearly observed (Figure 16) that the LMSR cycle has promoted a larger grain growth than the SRD method. Table 8 summarizes these differences. The measurement method consisted in measuring the primary alpha grain diameter. The measurement was taken repeatably with an average relative standard deviation of $18.4 \%$ (between $11 \%$ and $31 \%$ ) with a minimum of twentyfive measurements per micrograph. The average grain size difference of the observed points is $25 \%$. It is complex to dissociate the effect of static and dynamic grain

\begin{tabular}{c|c|c|c|c|c} 
Point & 1 & 2 & 3 & 4 & 5 \\
\hline$d_{\text {LMSR }}(\mu \mathrm{m})$ & 8.28 & 8.32 & 8.26 & 7.94 & 6.76 \\
$d_{S R D}(\mu \mathrm{m})$ & 5.70 & 6.41 & 6.14 & 6.32 & 5.10 \\
$\Delta d_{L M S R-S R D}(\%)$ & 31.1 & 22.9 & 25.7 & 20.4 & 24.5
\end{tabular}

Table 8: Relative grain size difference at some observation points

growth due to higher strain rates, as mentioned in section 1 . However, by comparing two areas which have undergone safe and very critical deformation, respectively point $1\left(\varepsilon_{\text {crit }}=0.77, \varepsilon_{\text {crit }}^{+}=0.25\right)$ and point $2\left(\varepsilon_{\text {crit }}=\varepsilon_{\text {crit }}^{+}=0\right)$, the grain size has grown larger in the safest conditions. One explanation is that the effect of time is enhanced with the deformation, but that the weight of time is more important than strain rate at this temperature.

\section{Conclusions}

The current process used by most FE software to implement SPF pressure designing does not take account of numerical issues which influence the forming time in an upward direction. The Gaussian theory for determining the maximum strain rate, which controls pressure algorithms, is a simple way to put aside the singular elements and significantly reduces the forming time. This new strategy was applied 
on an industrial part and the SPF was successful. The forming time of the tested industrial workpiece which is a particular case, was reduced by $67 \%$. For more classical geometries the time saving is around 30-50 \% according to the complexity of the geometry. The critical issues of SPF have been investigated:

- The thickness distribution is not impacted with the tested geometry.

- The grain growth is logically reduced, and heterogeneous grain growth is not enhanced due to higher strain rates. For this geometry the grain diameter is around $25 \%$ less than obtained by classical methods.

- The morphology of the grains is not impacted qualitatively, and no cavities are produced. This means that the deformation mechanisms remain superplastic.

This method has allowed improvement of the process while respecting the design specifications imposed by the aeronautics industry.

\section{Appendix}

\begin{tabular}{c|c|c} 
CASE & IF & THEN \\
\hline 1 & $\mathcal{R}^{n}<0.5$ & $P^{n, k+1}=2 P^{n, k}$ \\
1 & $0.5<\mathcal{R}^{n}<0.7$ & $P^{n, k+1}=1.5 P^{n, k}$ \\
1 & $0.7<\mathcal{R}^{n}<0.85$ & $P^{n, k+1}=1.25 P^{n, k}$ \\
2 & $0.85<\mathcal{R}^{n}<0.95$ & $P^{n+1}=1.15 P^{n, k}$ \\
2 & $0.95<\mathcal{R}^{n}<1.05$ & $P^{n+1}=P^{n, k}$ \\
3 & $1.05<\mathcal{R}^{n}<1.15$ & $P^{n, k+1}=P^{n, k} / 1.15$ \\
3 & $1.15<\mathcal{R}^{n}<1.3$ & $P^{n, k+1}=P^{n, k} / 1.25$ \\
3 & $1.3<\mathcal{R}^{n}<1.5$ & $P^{n, k+1}=P^{n, k} / 1.5$ \\
3 & $\mathcal{R}^{n}>1.5$ & $P^{n, k+1}=P^{n, k} / 2$
\end{tabular}

Table 9: Pressure regulation table - data 


\section{Acknowledgments}

The authors acknowledge AIRBUS Company for its financial support and the AUROCK Company where the forming experiments were performed. The authors are deeply grateful to Serge Tovar from Mines-Albi \& Institut Clément Ader for SEM analyses and the discussions regarding the microstructural aspects.

\section{References}

[1] E. Alabort, P. Kontis, D. Barba, K. Dragnevski, R. Reed, On the mechanisms of superplasticity in Ti6Al4V, Acta Materialia 105 (2016) 449-463.

[2] F. A. Mohamed, M. M. I. Ahmed, T. G. Langdon, Factors influencing ductility in the superplastic Zn-22 Pct Al eutectoid, Metallurgical Transactions A 8 (6) (1977) 933-938.

[3] R. B. Figueiredo, T. G. Langdon, Developing superplasticity in a magnesium AZ31 alloy by ECAP, Journal of Materials Science 43 (23-24) (2008) 7366-7371.

[4] H. Matsumoto, K. Yoshida, S.-H. Lee, Y. Ono, A. Chiba, Ti6Al4V alloy with an ultrafine-grained microstructure exhibiting low-temperaturehigh-strain-rate superplasticity, Materials Letters 98 (2013) 209-212.

[5] R. C. Gifkins, Grain-boundary sliding and its accommodation during creep and superplasticity, Metallurgical Transactions A 7 (8) (1976) 1225-1232.

[6] T. G. Langdon, An Analysis of Flow Mechanisms in High Temperature Creep and Superplasticity, Materials Transactions 46 (9) (2005) 1951-1956.

[7] V. Velay, H. Matsumoto, V. Vidal, A. Chiba, Behavior modeling and microstructural evolutions of Ti6Al4V alloy under hot forming conditions, International Journal of Mechanical Sciences 108-109 (2016) 1-13.

[8] M. Bellet, Modelisation numerique du formage superplastique de toles, Ph.D. thesis, 1988. 
[9] D. Woodford, Strain rate sensitivity as a measure of ductility, ASM trans. 62 (1969) 291-293.

[10] D. Garriga-Majo, R. J. Paterson, R. V. Curtis, R. Said, R. D. Wood, J. Bonet, Optimization of the superplastic forming of a dental implant for bone augmentation using finite element simulations, Dental Materials 20 (5) (2004) 409-418.

[11] X. D. Ding, H. M. Zbib, C. H. Hamilton, A. E. Bayoumi, On the optimization of superplastic blow-forming processes, Journal of Materials Engineering and Performance 4 (4) (1995) 474-485.

[12] M. A. Nazzal, M. K. Khraisheh, B. M. Darras, Finite Element Modeling and Optimization of Superplastic Forming Using Variable Strain Rate Approach, Journal of Materials Engineering and Performance 13 (6) (2004) 691-699.

[13] L. C. Chung, J.-H. Cheng, The analysis of instability and strain concentration during superplastic deformation, Materials Science and Engineering: A 308 (12) (2001) 153-160.

[14] L. C. Chung, J.-H. Cheng, Fracture criterion and forming pressure design for superplastic bulging, Materials Science and Engineering: A 333 (1-2) (2002) $146-154$.

[15] E. Hart, Theory of the tensile test, Acta Metallurgica 15 (2) (1967) 351-355.

[16] C. Robert, Contribution to the numerical simulation of sheet metal forming proceses - application to incremental sheet forming and superplastic forming., Ph.D. thesis, Arts et Métiers ParisTech, 2009.

[17] J. St. Doltsinis, J. Luginsland, S. Nölting, Some developments in the numerical simulation of metal forming processes, Engineering Computations 4 (4) (1987) $266-280$.

[18] Y. Xiang, S. Wu, Numerical simulation of cavity damage evolution in superplastic bulging process, Journal of Materials Processing Technology 116 (2-3) (2001) 224-230. 
[19] Y.-M. Hwang, H. Lay, J. Huang, Study on superplastic blow-forming of 8090 AlLi sheets in an ellip-cylindrical closed-die, International Journal of Machine Tools and Manufacture 42 (12) (2002) 1363-1372.

[20] L. Carrino, G. Giuliano, G. Napolitano, A posteriori optimization of the forming pressure in superplastic forming processes by the finite element method, Finite Elements in Analysis and Design 39 (11) (2003) 1083-1093.

[21] T. Ahmed, H. Rack, Phase transformations during cooling in $\alpha+\beta$ titanium alloys, Materials Science and Engineering: A 243 (1) (1998) 206-211. 